\title{
Pore-based ridge reconstruction for fingerprint recognition
}

\author{
Maurício Pamplona Segundo, Rubisley de Paula Lemes \\ Intelligent Vision Research Lab, Department of Computer Science, Federal University of Bahia, Brazil \\ $\{$ mauricio, rubisley\} @dcc.ufba.br
}

\begin{abstract}
The use of sweat pores in fingerprint recognition is becoming increasingly popular, mostly because of the wide availability of pores, which provides complementary information for matching distorted or incomplete images. In this work we present a fully automatic pore-based fingerprint recognition framework that combines both pores and ridges to measure the similarity of two images. To obtain the ridge structure, we propose a novel pore-based ridge reconstruction approach by considering a connect-the-dots strategy. To this end, Kruskal's minimum spanning tree algorithm is employed to connect consecutive pores and form a graph representing the ridge skeleton. We evaluate our framework on the PolyU HRF database, and the obtained results are favorably compared to previous results in the literature.
\end{abstract}

\section{Introduction}

Most commercial Automatic Fingerprint Identification Systems (AFIS) rely on Level 1 (i.e. global characteristics - ridge flow and pattern type) and Level 2 (i.e. local features - minutiae and ridge skeleton) features to distinguish genuine and impostor individuals [5]. They achieve high recognition accuracy, but depend on image quality to do so. For this reason, the use of Level 3 features (e.g. sweat pores and incipient ridges) as a complementary information for matching has attracted the attention of many researchers in the literature. Among these features, the use of pores stood out as a promising way to improve the recognition performance $[5,7,8,10,13-15]$.

A pore-based AFIS usually has three main stages: (1) pore detection, (2) pore correspondence establishment and (3) similarity evaluation. Pore detection is a challenging task since pores vary widely in size and shape (see Figure 1). Some initial works $[5,15]$ did not adapt themselves to such variations and, as a consequence, faced large numbers of false positives and false negatives. Other works $[13,14]$ addressed adaptability, but at the cost of using computationally expensive techniques, such as ridge reconstruction and frequency estimation. Recently, Lemes et al. [7] proposed an accurate method, named Dynamic Pore Filtering (DPF), that efficiently adjusts itself to pore variations without requiring these time-consuming calculations.

To establish pore correspondences, global alignment [5] or local pairwise correspondences [7, 8, 10,14] can be considered. The second one has become increasingly popular because keypoint description and matching is a very hot topic in computer vision research nowadays. There is a number of descriptors that can be used to characterize pores (e.g. sparse representation [8], SIFT [10], DAISY [7]), and pore correspondences can be obtained by matching those descriptors. In this stage, an outlier filtering step is usually necessary to eliminate wrong correspondences.

Finally, a matching score is computed based on pore correspondences and then employed to decide whether the match is genuine or not. Typical score choices are the number of correspondences $[8,14]$ or the average reprojection error [5]. This type of matching, however, does not consider how pores are related to each other, and consequently misses important pieces of information which could help the decision making process.

In this work, we present a fully automatic pore-based fingerprint recognition framework. Unlike other works in the literature, we connect consecutive pores and use this information for matching as well. To perform the connecting task, as our first contribution, we propose a novel ridge reconstruction approach based on a connect-the-dots strategy. To this end, pores are interpreted as vertices of a graph and Kruskal's algorithm [6] is employed to find the minimum spanning tree that connects them. Then, as our second contribution, we present a pore-based matching approach that also takes the connection between pores in consideration.

\section{Pore detection}

Since we use pores for both ridge reconstruction and fingerprint matching, we need a fast and accurate approach to detect them. To fulfill such requirements, we use DPF [7].

DPF has two main stages: (1) pore size estimation and (2) pore classification. To estimate the pore size, for each pixel with value above a global threshold (i.e. bright pixel), the closest neighbor with value under the global threshold 
(i.e. dark pixel) is found in four directions: up, down, left and right. The distance to these neighbors is then used to estimate a global radius for pores (i.e. average valley width). This global radius defines the size of a mask that is centered on each bright pixel, which is then used to estimate a local threshold and a local radius. In the classification stage, a circle around each bright pixel with local radius is used to determine whether it is a pore or not based on three conditions: the proportion of bright pixels in the circle must be under $40 \%$; the number of transitions between bright and dark pixels must be smaller than two; and the average pixel value in the circle must be below the local threshold.

As may be seen in Figure 1(a), using the average valley width to estimate global and local radius may return a similar pore size in very different situations. We may also observe that ridges seem to be a better clue for pore size estimation, so we estimate global and local radius using the average ridge width. With this small change, DPF adapts even better to pore variations, as illustrated in Figure 1(b), and the detection accuracy is improved.
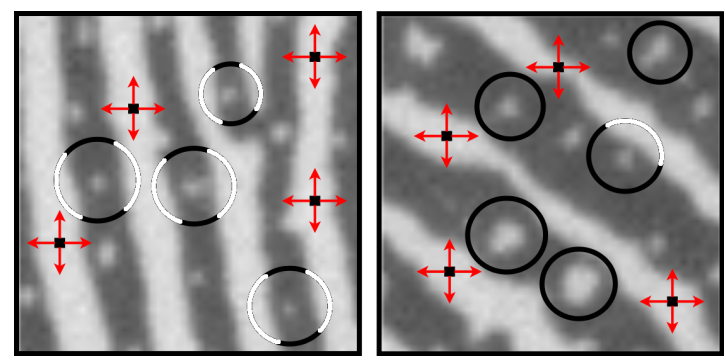

(a)
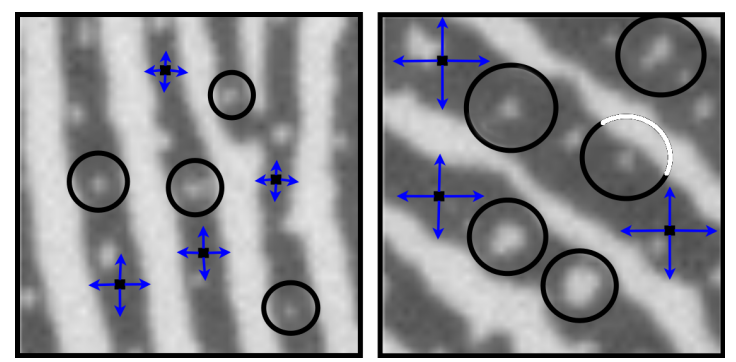

(b)

Figure 1. Illustration of the pore size estimation step based on (a) average valley width and (b) average ridge width.

\section{Ridge reconstruction}

Ridge reconstruction is an important step in different AFIS and consists in locating ridges and representing them through their skeleton. Ridges can be used in different fingerprint analysis, such as matching [2], minutiae extraction [5] and image compression [4], and previous works explored different ideas to reconstruct them: line tracing [12], ridge points detection [3], image binarization and thin- ning [1] and so on. We propose a novel ridge reconstruction approach based on a connect-the-dots strategy. The objective is to establish connections between pores and, by doing so, to obtain the ridge skeleton. To this end, we build a complete weighted graph using pores as vertices and then use Kruskal's algorithm [6] to find the minimum spanning tree that discards non-ridge edges. To the best of our knowledge, this is the first attempt to reconstruct ridges using pores.

First we interpret an input fingerprint image $I$ as a weighted graph $G=(V, E)$. Every vertex $u \in V$ represents a pore and has coordinates $\left(x_{v}, y_{v}\right)$. Every edge $(u, v) \in E$ represents a line between $\left(x_{u}, y_{u}\right)$ and $\left(x_{v}, y_{v}\right)$ in $I$, and has a weight $w_{u v}$ computed as in Equation 1:

$$
w_{u v}=\alpha|L|+\sum_{p \in L} \frac{I\left(x_{p}, y_{p}\right)^{2}}{255 \max \left(\left|x_{p}-\bar{x}_{L}\right|,\left|y_{p}-\bar{y}_{L}\right|\right)}
$$

where $L$ is the set of pixels that form a line between $u$ and $v$, as illustrated in Figure 2, $I\left(x_{p}, y_{p}\right)$ is the grayscale value for the pixel $p$, and $\left(\bar{x}_{L}, \bar{y}_{L}\right)$ is the midpoint in $L$, also illustrated in Figure 2.

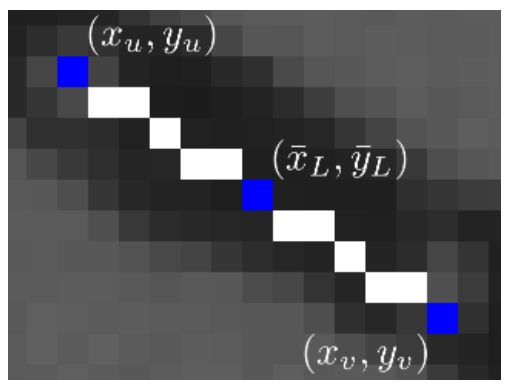

Figure 2. Line of pixels between pores $u$ and $v$. Pixels corresponding to $u, v$ and line midpoint are shown in blue.

Equation 1 is formulated in a way that penalizes edges that contain bright pixels (i.e. $\left.\frac{I\left(x_{p}, y_{p}\right)^{2}}{255}\right)$ in the center of the line (i.e. $\left.\frac{1}{\max \left(\left|x_{p}-\bar{x}_{L}\right|,\left|y_{p}-\bar{y}_{L}\right|\right)}\right)$ and/or edges that are too long (i.e. $\alpha|L|$ ). The value of $\alpha$ is a parameter of the approach that affects the importance of the edge length in the reconstruction process. Details regarding this and other subsequent parameters are presented in Section 5.3. In this formulation, edges connecting consecutive pores tend to have lower weights than edges crossing valleys since they are both dark and short.

Although $G$ could be constructed as a complete graph, many edges are too long and would never be part of the final ridge structure. To save processing power, we discard edges if their length are greater than $\beta \bar{d}$, where $\bar{d}$ is the average distance between two consecutive pores and $\beta$ is a parameter that defines how long edges can get. Since the ridge structure is not yet available, $\bar{d}$ cannot be precisely estimated. However, we assume a uniform grid spacing between pores to approximate its value, as in Equation 2. 


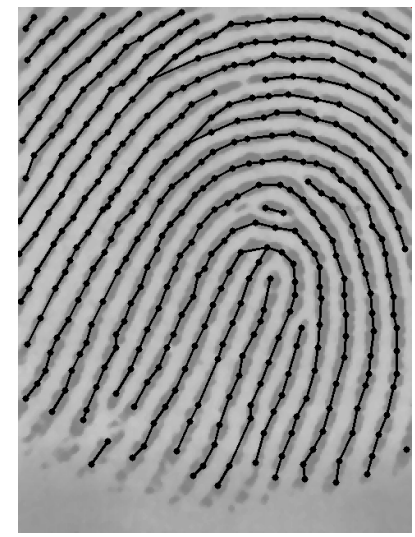

(a) GROUND TRUTH

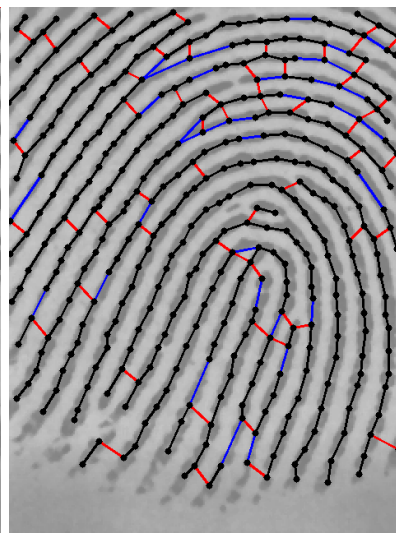

(b) DR: $90.1 \%$ / FDR: $15.2 \%$

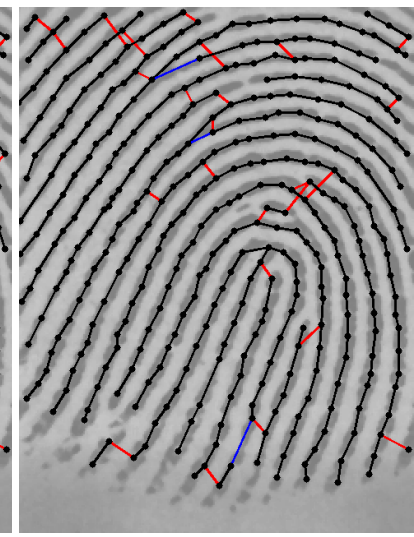

(c) DR: $99.1 \%$ / FDR: $6.7 \%$

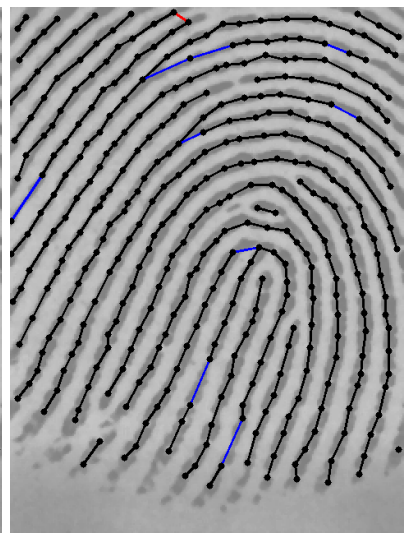

(d) DR: $97.4 \%$ / FDR: $0.3 \%$

Figure 3. Results of the proposed pore-based ridge reconstruction approach: (a) ground truth, ridge structure after running Kruskal's algorithm in both (b) raw and (c) enhanced images; and (d) ridge structure after edge filtering. False positives are shown in red and false negatives are shown in blue. Edges in black were correctly detected by the proposed approach.

$$
\bar{d} \approx\left(\sqrt{\frac{|V|}{I_{\text {rows }} * I_{\text {cols }}}}\right)^{-1}
$$

Finally, we run Kruskal's algorithm and take the resulting tree as the ridge structure. A pseudocode for Kruskal's algorithm is provided in Algorithm 11, and, as may be observed, it is a greedy search that takes low-weight edges first and removes all cycles in the graph. As edges between consecutive pores have lower weights, they are frequently taken as part of the minimum spanning tree.

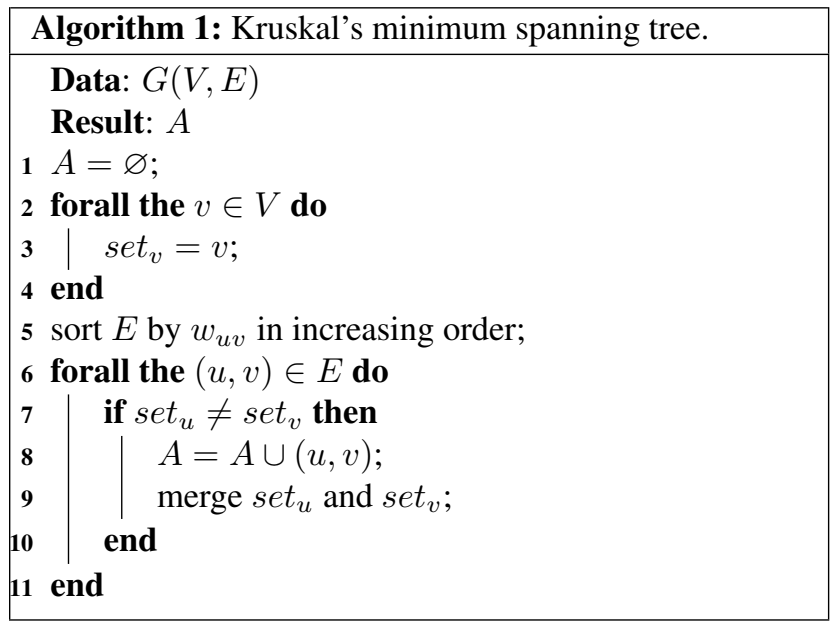

An example of the ridge reconstruction result is shown in Figure 3(b). As may be seen, this initial result is very far from ideal, which is mainly caused by noise and contrast changes in the fingerprint image. To reduce effects of these artifacts, we apply the Contrast Limited Adaptive Histogram Equalization (CLAHE) [11] to enhance image con- trast. Using CLAHE, however, may end up sharpening the noise (see Figure 4(b)). To avoid this, we apply a $3 \times 3$ median filter to the input image before applying CLAHE. The resulting image is shown in Figure 4(c), and the improvement in ridge reconstruction results when using enhanced images is presented in Figure 3(c).

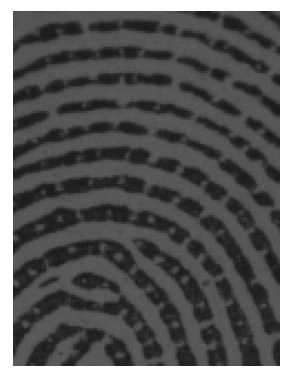

(a) ORIGINAL

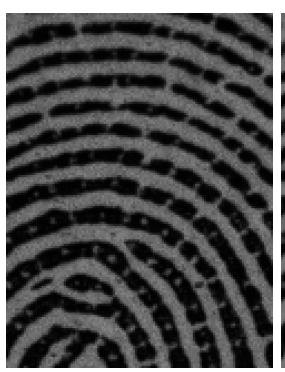

(b) CLAHE

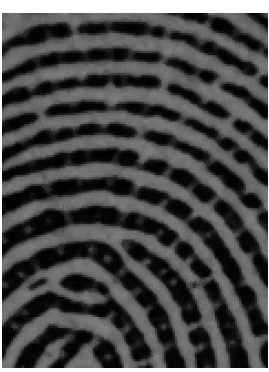

(c) MEDIAN+CLAHE
Figure 4. Fingerprint image enhancement: (a) original image, and result for CLAHE-based image enhancement (b) without and (c) with noise filtering.

After image enhancement, reconstruction results are much more visually sound, but many false positives (i.e. edges that should not be part of the ridge structure) are still present. Most of them are selected in Algorithm 11 because the resulting tree must also be a connected graph, which is not the case for a graph of a fingerprint image (see Figure 3(a)). In addition, Kruskal's algorithm has no information on how ridges are arranged, so it may over-connect vertices and/or select intersecting edges.

To overcome these problems, we specified some criteria to filter out false positives. First we created two rules to add an edge $(u, v)$ to $A$ in Algorithm 11: (1) $(u, v)$ cannot intersect any other edge already in $A$; and (2) neither $u$ or $v$ can already have two or more edges. The second rule 
prevents us from properly representing bifurcations in the ridge structure, but it also prevents forming non-existing bifurcations, which is a common mistake (see Figure 3(c)). Then, any edge whose weight is greater than $\mu_{A}+\gamma \sigma_{A}$ is discarded, where $\mu_{A}$ is the average weight for all edges in $A, \sigma_{A}$ if the standard deviation, and $\gamma$ is a parameter that defines how bright an edge must be to be discarded.

The result of this edge filtering step is shown in Figure 3(d). Both detection rate (DR) and false discovery rate (FDR) are also presented in Figures 3(b)-3(d). DR represents the percentage of edges in the ground truth that were successfully found, and FDR represents the proportion of false positives among the edges in $A$. As may be observed, DR decreased about $1.7 \%$ after edge filtering, but FDR decreased even more, to $0.3 \%$. The final ridge structure is very accurate, and is further used for matching in Section 4. A more detailed analysis of the reconstruction performance is presented in Section 5.3.

\section{Fingerprint matching}

Most pore-based fingerprint matching systems rely on finding corresponding pores and filtering outliers to measure the similarity between two images $[8,16]$. Following this idea, we extract a descriptor for each pore using the Scale-Invariant Feature Transform (SIFT) [9] with scale $s$ (i.e. details on parameters are given in Section 5.4). Then, we look for bidirectional correspondences between pores from gallery and probe images (i.e. the ratio between the closest and the second closest descriptor distances must be smaller than $r$ - see Section 5.4) and use these correspondences to compute the matching score. The score considers both ridge structure and consistency of corresponding points to measure the similarity, as in Equations 3-5:

$$
\begin{gathered}
\text { score }=\sum_{i=1}^{|C|} f(i) \\
f(i)= \begin{cases}g(i), & \text { if } \exists j \neq i \mid \exists u_{i}^{A} \ldots u_{j}^{A} \wedge \exists u_{i}^{B} \ldots u_{j}^{B} \\
0, & \text { otherwise }\end{cases} \\
g(i)=\sum_{j=1}^{|C|} \frac{1}{1+\left|\left\|u_{i}^{A}-u_{j}^{A}\right\|-\left\|u_{i}^{B}-u_{j}^{B}\right\|\right|}
\end{gathered}
$$

where $C=\left\{\left(u_{1}^{A}, u_{1}^{B}\right), \ldots,\left(u_{|C|}^{A}, u_{|C|}^{B}\right)\right\}$ is the set of corresponding pores, $A$ and $B$ are ridge graphs for gallery and probe images respectively, $u \ldots v$ is a path of edges connecting $u$ to $v$, and $\|u-v\|$ is the Euclidean distance between the coordinates of $u$ and $v$. Equation 4 takes the ridge structure in consideration by discarding corresponding pores that are not connected to at least one other pair of corresponding pores through a path in both $A$ and $B$. Thus, only pores in ridges with multiple correspondences are selected, and the remaining ones are considered false positives. Equation 5 verifies if the distance between two pores in the gallery image is similar to the distance between their corresponding pores in the probe image, and consequently measures the consistency of pores selected in Equation 4. Even though fingerprint distortion affect $g(i)$, a global analysis of the consistency is preferred to avoid local maxima.

\section{Experimental results}

\subsection{Database}

In this work we use The Hong Kong Polytechnic University High-Resolution-Fingerprint ${ }^{1}$ (PolyU HRF) database [8,14-16]. This database has one training set and two testing sets, and its images have 1200 dpi resolution. The training set has 210 images of 35 different subjects (i.e. 6 images per subject acquired in two different sessions 3 images per session). Both testing sets, named DBI and DBII, have 1480 images from 148 different subjects (i.e. 10 images per subject acquired in two different sessions -5 images per session). Images in training and DBI sets have $320 \times 240$ pixels (i.e. partial fingerprints), while DBII has images with $640 \times 480$ pixels (i.e. full-size fingerprints). The PolyU HRF database also contains a subset of DBI images that contains the ground truth coordinates of pores (i.e. 30 images with an average of 425 pores).

\subsection{Pore detection results}

In this experiment, we use the subset of the PolyU HRF database that contains the ground truth coordinates of pores to compute DR and FDR. We compare DPF's performance with four state-of-the-art approaches, as may be seen in Table 1 (i.e. results for Jain et al. [5] and Zhao et al. [14, 15] were reported in Teixeira et al.'s work [13]).

Table 1. DR and FDR for pore detection.

\begin{tabular}{|l||c|c|}
\hline Method & DR & FDR \\
\hline \hline Jain et al. [5] & $75.9 \%$ & $23.0 \%$ \\
\hline Zhao et al. $[15]$ & $80.8 \%$ & $22.2 \%$ \\
\hline Zhao et al. $[14]$ & $84.8 \%$ & $17.6 \%$ \\
\hline Teixeira et al. $[13]$ & $86.1 \%$ & $8.6 \%$ \\
\hline DPF $[7]$ & $83.5 \%$ & $9.9 \%$ \\
\hline Enhanced DPF & $90.8 \%$ & $11.1 \%$ \\
\hline
\end{tabular}

Table 1 shows that enhanced DPF and Teixeira et al.'s work [13] have comparable results and both outperform other reported works. However, DPF requires less computational resources since it does not require any timeconsuming and quality-dependent processing steps, such as ridge reconstruction, ridge map estimation, ridge orientation estimation and/or frequency estimation.

\footnotetext{
${ }^{1}$ http://www4.comp.polyu.edu.hk/biometrics/HRF/HRF_old.htm
} 


\subsection{Ridge reconstruction results}

To evaluate the accuracy of the proposed ridge reconstruction approach, we also use the subset of the PolyU HRF database that contains the ground truth coordinates of pores. We extended the ground truth to include the ridge structure of these images by manually marking valid edges between annotated pores. An example of the extended ground truth is shown in Figure 3(a).

In our experiments, we run our approach using ground truth pores and then compare the obtained reconstruction to the ground truth graph. If an edge is present in the reconstruction result but is not in the ground truth, it is considered a false positive. If it is in the ground truth but is not in the reconstruction result, it is considered a false negative. We count the number of false positives and false negatives over the entire test set to obtain both DR and FDR.

This experiment was repeated several times, and different values for $\alpha, \beta$ and $\gamma$ were considered. $\alpha$ was tested over the range $[0,255]$ with step size $1, \beta$ over the range $[1.0,5.0]$ with step size 0.5 , and $\gamma$ over the range $[1.0,3.0]$ with step size 0.1 . The best performance was obtained with $\alpha=16, \beta=2.5$ and $\gamma=1.7$, and the results are shown in Table 2. As may be observed, image enhancement and edge filtering considerably reduce the number of false positives and false negatives.

Table 2. DR and FDR for ridge reconstruction.

\begin{tabular}{|l||c|c|}
\hline Modality & DR & FDR \\
\hline \hline Raw image & $92.8 \%$ & $13.1 \%$ \\
\hline Enhanced image & $95.3 \%$ & $10.8 \%$ \\
\hline Enhanced image + edge filtering & $94.1 \%$ & $3.9 \%$ \\
\hline
\end{tabular}

Finally, we use the best configuration found to reconstruct fingerprint ridges using automatically detected pores. This task is more challenging since we may have missing pores, pores detected multiple times, or false positives from pore detection. Figure 5(a) illustrates these problems and shows that the proposed approach is still able to provide a meaningful interpretation of the ridge structure, although reconstruction results are better when the pore detection is more accurate (see Figure 5(b)).

\subsection{Matching results}

Both pores and ridges were automatically extracted for matching. The training set of the PolyU HRF database was used to find the parameter values that maximize the recognition accuracy of the proposed approach. SIFT scale $s$ was tested over the range $[1,8]$ with step size 1 , and thresholding ratio $r$ over the range $[0.1,1.0]$ with step size 0.1 . The highest recognition accuracy in the training set was obtained when $s=4$ and $r=0.8$. These were then used unchanged in our experiments on DBI and DBII.

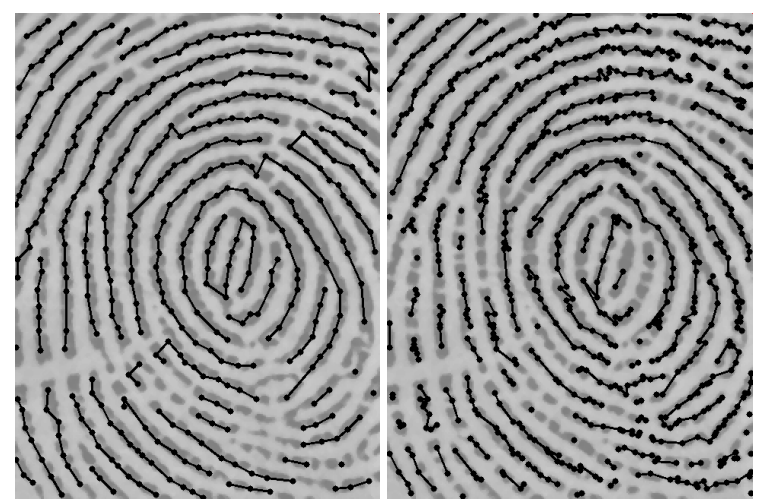

(a)

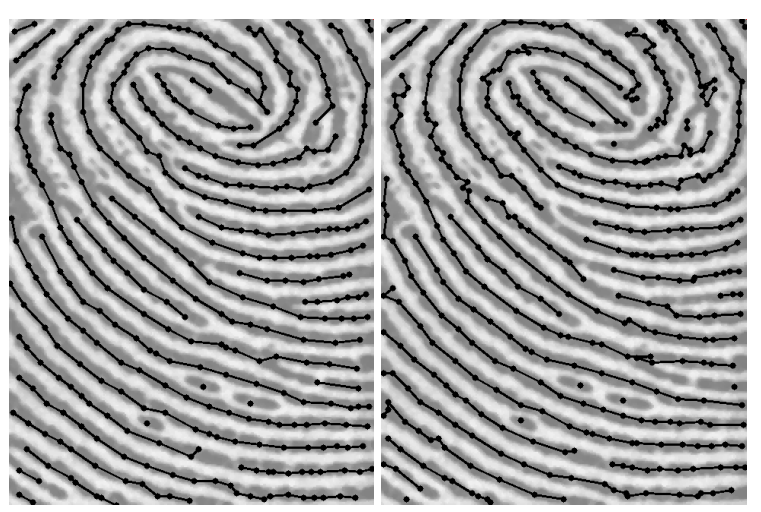

(b)

Figure 5. Pore-based ridge reconstruction results using (left) ground truth pores and (right) automatically detected pores.

We used the same methodology proposed by Liu et $a l$. [8] to evaluate our matching performance on DBI and DBII. First we match images of the same subject in different sessions to obtain the genuine score set (i.e. 3,700 combinations). Then, we match the first image of each subject in the first session to the first image of all other subjects in the second session and obtain the impostor score set (i.e. 21,756 combinations). The obtained scores are then used to compute the Receiver Operating Characteristic (ROC) curve, which combines the False Rejection Rate (FRR) and the False Acceptance Rate (FAR). The resulting curves for DBI and DBII are shown in Figure 6. Results for DBII are better than for DBI, as expected, since DBII images have full-size fingerprints.

We also compared our results to other pore-based fingerprint recognition works in the literature. Table 3 shows the Equal Error Rate (EER) for DBI and DBII using Minutia and Pore alignment with Iterative Closest Points (MICPP) [5], Direct Pore matching (DP) [16], Sparse Representation-based Direct Pore matching (SRDP) [8] and the proposed approach (i.e. results for MICPP, DP and SRDP were reported in Liu et al.'s work [8]). As may be observed, we reduced EER for DBI and DBII in more than 


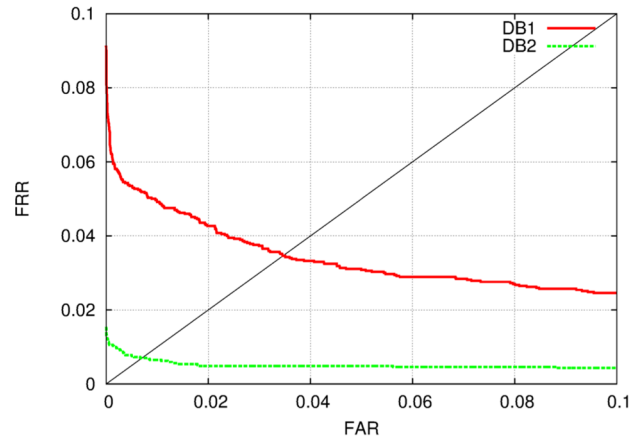

Figure 6. ROC curves of the proposed approach for DBI and DBII.

$40 \%$ and $20 \%$, respectively, when compared to the best results in the literature. These results show the potential of using pore connections in pore-based fingerprint matching and also validate the proposed pore-based ridge reconstruction approach.

Table 3. EER for fingerprint matching using DBI and DBII.

\begin{tabular}{|l||c|c|}
\hline Approach & DBI & DBII \\
\hline \hline MICPP [5] & $30.45 \%$ & $7.83 \%$ \\
\hline DP [16] & $15.42 \%$ & $7.05 \%$ \\
\hline SRDP [8] & $6.59 \%$ & $0.97 \%$ \\
\hline Proposed & $3.74 \%$ & $0.76 \%$ \\
\hline
\end{tabular}

\section{Conclusion}

We propose a novel strategy to reconstruct fingerprint ridges, which consists in connecting consecutive pores through edges to obtain the ridge skeleton. We also propose an approach to automatically perform this reconstruction based on Kruskal's minimum spanning tree algorithm. To the best of our knowledge, this is the first attempt to reconstruct ridges using pores. Our approach detects $94.1 \%$ of the ground truth edges with only $3.9 \%$ of false detections.

Then, we incorporate this ridge information in the porebased matching stage in order to discard wrong pore correspondences. We achieve a $3.74 \%$ EER on DBI and a $0.76 \%$ EER on DBII, which outperform other works in the literature. These results validates the proposed ridge reconstruction approach, showing that the obtained ridge structure is suitable for fingerprint recognition.

The entire recognition framework does not rely on quality-dependent processing methods, making it well suited for distorted or damaged fingerprint images. As a future work, we intend to investigate this issue and other options for graph-based ridge matching (e.g. graph isomorphism and maximum common subgraph).

\section{Acknowledgments}

We would like to thank the financial support from the National Counsel of Technological and Scientific Develop- ment (CNPq) and The Hong Kong Polytechnic University for allowing access to the PolyU HRF database.

\section{References}

[1] S. Chikkerur, A. N. Cartwright, and G. V. Fingerprint enhancement using STFT analysis. Pattern Recognition, 40(1):198-211, 2007. 2

[2] H. Choi, K. Choi, and J. Kim. Fingerprint matching incorporating ridge features with minutiae. IEEE TIFS, 6(2):338345, 2011. 2

[3] C. Domeniconi, S. Tari, and P. Liang. Direct gray scale ridge reconstruction in fingerprint images. In IEEE ICASSP, pages 2941-2944, 1998. 2

[4] M. Gokmen, I. Ersoy, and A. Jain. Compression of fingerprint images using hybrid image model. In IEEE ICIP, pages 395-398, 1996. 2

[5] A. Jain, Y. Chen, and M. Demirkus. Pores and ridges: Highresolution fingerprint matching using level 3 features. IEEE TPAMI, 29(1):15-27, 2007. 1, 2, 4, 5, 6

[6] J. B. Kruskal. On the Shortest Spanning Subtree of a Graph and the Traveling Salesman Problem. Proceedings of the American Mathematical Society, 7(1):48-50, 1956. 1, 2

[7] R. Lemes, M. Pamplona Segundo, O. Bellon, and L. Silva. Dynamic pore filtering for keypoint detection applied to newborn authentication. In IEEE ICPR, pages 1698-1703, 2014. 1, 4

[8] F. Liu, Q. Zhao, D. Zhang, and D. Zhang. Fingerprint pore matching based on sparse representation. In IEEE ICPR, pages 1630-1633, 2010. 1, 4, 5, 6

[9] D. G. Lowe. Object recognition from local scale-invariant features. In IEEE ICCV, pages 1150-1157, 1999. 4

[10] S. Malathi and C. Meena. Improved partial fingerprint matching based on score level fusion using pore and sift features. In IEEE PACC, pages 1-4, 2011. 1

[11] S. Pizer, R. Johnston, J. Ericksen, B. Yankaskas, and K. Muller. Contrast-limited adaptive histogram equalization: speed and effectiveness. In Proc. 1st Conference on Visualization in Biomedical Computing, pages 337-345, 1990. 3

[12] Y. Qi. Fingerprint ridge line reconstruction. In Intelligent Information Processing II, volume 163 of IFIP, pages 211220. Springer US, 2005. 2

[13] R. Teixeira and N. Leite. Improving pore extraction in high resolution fingerprint images using spatial analysis. In IEEE ICIP, pages 4962-4966, 2014. 1, 4

[14] Q. Zhao, D. Zhang, L. Zhang, and N. Luo. Adaptive fingerprint pore modeling and extraction. Pattern Recognition, 43(8):2833-2844, 2010. 1, 4

[15] Q. Zhao, D. Zhang, L. Zhang, and N. Luo. High resolution partial fingerprint alignment using pore-valley descriptors. Pattern Recognition, 43(3):1050-1061, 2010. 1, 4

[16] Q. Zhao, L. Zhang, D. Zhang, and N. Luo. Direct pore matching for fingerprint recognition. In Advances in Biometrics, volume 5558 of $L N C S$, pages 597-606. Springer Berlin Heidelberg, 2009. 4, 5, 6 